\title{
A Framework for Process Inspection of Metal Additive Manufacturing
}

\author{
Chih-Kun Cheng, ${ }^{1 *}$ Frank Liou, ${ }^{2}$ Yi-Chien Cheng, ${ }^{3}$ and Sheng-Chih Shen ${ }^{3}$
}

\author{
${ }^{1}$ Department of Electrical Engineering, Far East University, Taiwan \\ ${ }^{2}$ Department of Mechanical Engineering, Missouri University of Science and Technology, USA \\ ${ }^{3}$ Department of Systems and Naval Mechatronic Engineering, National Cheng Kung University, Taiwan
}

(Received June 6, 2018; accepted October 24, 2018)

Keywords: additive manufacturing, process inspection, process parameters

In this paper, we propose a process inspection framework for metal additive manufacturing (AM) processes. AM, also known as 3D printing, is the process of joining materials to make objects on the basis of 3D model data and is envisioned to play a strategic role in maintaining economic and scientific dominance. Different from conventional manufacturing methods, the AM process is a point-by-point and layer-by-layer manufacturing. Thus, there are many opportunities to generate a process error that can cause quality issues in an AM part. A systematic AM process inspection is needed to yield acceptable performance of the part. The critical parameters that may affect the part quality are identified before processing, during processing, and after processing. The framework of the initial AM process inspection is presented. By using basic sensors, such as a microhardness tester and profilometer, we can obtain critical information about an additive manufactured part.

\section{Introduction}

Rapid prototyping and 3D printing emerged in the United States in the 1980s, and many innovations concerning new materials and new manufacturing processes as well as new equipment have been constantly developed. Rapid prototyping technology has been in existence for many years. American Society for Testing and Materials (ASTM) formalized the term "additive manufacturing" (AM) technology in 2009. AM technology can quickly convert a computer model of a part into a net-shape physical part. The heating renders it into a molten state and then various layers are printed to produce a workpiece. 3D printing technology is now widely used in various industries. At present, there are several commercial metal AM technologies, as follows.

Selective laser melting (SLM) is an AM technique in which the metal to be processed is in a powder form that is spread over a build platform as a thin layer using a wiper. A fiber laser melts the fine metal powder into metal parts. A computer-aided design (CAD) 3D file and an SLM planner dictate where melting will occur. This process must be completed in a workspace

*Corresponding author: e-mail: n2888122@yahoo.com.tw

https://doi.org/10.18494/SAM.2019.2106 
filled with inert gas under precision-controlled pressure. Common materials include stainless steel, cobalt-chromium alloy, titanium metal, and aluminum. Although the strength of the finished product is slightly inferior, the disadvantage that the structure at the welding point is easily damaged can be avoided because the welding procedure is omitted.

Selective laser sintering (SLS) is similar to SLM in that the characteristics of the resulting objects are significantly different. Whereas SLM is only accomplished by melting followed by solidification by cooling, SLS is formed by sintering, and the grain structure and porosity of the finished product are different from those of the original powder. SLS can also yield non-fully dense metals, but it is mostly used for plastics. Since this paper is about metals, we will not discuss it further here.

Directed energy deposition (DED) covers a range of terminologies: laser-engineered net shaping, directed light fabrication, and direct metal deposition. DED uses the material in wire or powder form. It utilizes focused energy (either an electron beam or a laser beam) to fuse materials by melting as the material is being deposited from any angle. ${ }^{(1)}$ The nozzle through which the material is deposited is typically mounted on a five-axis arm. Then the deposited material is melted by a laser or electron beam. The material is added layer by layer and solidified, creating or repairing new material features on the existing object.

These technologies were used extensively in aerospace and other industries. For some period of time, some companies have been experimenting with laser welding equipment on motion stages and using them to construct near-net-shape geometries.

AM technology offers a distinct manufacturing method that can provide a fast turn-around method of manufacture directly from a CAD model. For example, the SLM technology allows the production of parts from various metal materials by selectively fusing metallic powder material. The resulting static mechanical properties are generally comparable to those of conventional process materials, such as stainless steel and hot-work steel, ${ }^{(2,3)}$ aluminum, ${ }^{(4-6)}$ titanium, ${ }^{(7)}$ and Ni-based materials. ${ }^{(8,9)}$

AM can be used to quickly fabricate and join parts, fixtures, and tools with complex geometry. However, one major issue of AM is the lack of control of the part quality. The challenges in the AM process quality include consistent manufacture of the correct part geometry and of parts with the intended material properties. These inconsistencies are caused partially by the very high heating and cooling rates, and the resulting nonequilibrium microstructures and high residual stresses. The quality issue can be further compromised by inappropriate material input and process parameter selections. These metal AM processes have high heating and cooling rates, which results in excellent adhesion through the metallurgical bond between the coating material and the substrate. To investigate the process parameters, extensive testing, such as tensile and fatigue tests, of the produced samples may be needed. However, these tests are very time-consuming and costly. In this paper, we outline a systematic strategy for AM process inspection and summarize the initial test strategy using nondescriptive methods, such as hardness and surface roughness tests, to provide a quick initial assessment of the produced samples.

In this paper, we describe the interrelationships among various parameters. Depending on the applications, the identified parameters can be used for online or offline inspections using 
various sensor technologies. The online inspection is technically more challenging. Thus offline inspection may be the first step in the calibration of the parameters. For example, from the sensors, such as a microhardness tester and profilometer, we can obtain the hardness and surface finish of the part. Along with the process parameters and the interrelationship discussed in this paper, we can fine-tune these parameters. Once the nominal set of parameters are set, we can then proceed with online sensors for in situ process monitoring and control. For example, various parameters related to energy density can be monitored by online sensors, and control can be carried out for abnormal situations, such as when depositing material on an edge or a corner. The parameters can be adjusted in accordance with the energy density equation.

\section{Effect of Input Parameters on AM Processing}

Many parameters affect the AM process and they can be divided into four categories: (1) laser-related parameters such as laser power, beam quality, spot size, laser frequency, and angle of incidence; (2) parameters related to scanning, such as scanning speed, scanning pitch, scanning angle, and scanning pattern; (3) powder-related parameters, such as powder material, grain shape, surface morphology, particle size and distribution, bulk density, and layer thickness; and (4) environmental parameters, such as powder temperature and uniformity, flow field distribution, and oxygen concentration.

\section{Energy Density}

The above phenomena can be better expressed as energy density. Energy density is the amount of energy stored in a unit volume: ${ }^{(3)}$

$$
E-\text { density }=\frac{P_{\text {Laser }}}{v_{\text {scan }} \cdot h_{s} \cdot t_{\text {Layer }}}\left(\mathrm{J} / \mathrm{mm}^{3}\right)
$$

In Eq. (1), $P_{\text {Laser }}$ is the laser power, $v_{\text {scan }}$ is the scanning speed, $h_{s}$ is the scanning distance, and $t_{\text {Layer }}$ is the thickness of the powder layer.

The energy density affects many aspects of metal deposition, including the porosity of the part. Insufficient energy density or too much energy density will yield poor deposition, such as porosity. Insufficient energy will result in lack of fusion and poor part quality. Too much energy will cause a balling effect or a keyhole effect, and thus may also result in porosity and poor surface quality. Inspecting the surface finish and hardness of the part may reveal whether the energy density is properly applied.

\section{Surface Roughness}

Surface roughness is a component of surface texture. It is measured by the deviation in the direction of the normal vector of a real surface from its ideal form. Surface roughness is also a local property of a part. However, since it is a nondestructive testing method, there is much 
data that can show the real quality of the additive manufactured parts. There are many different roughness parameters being used, but amplitude parameters or $R a$ is a common representation. Amplitude parameters describe the surface on the basis of the vertical deviations of the roughness profile from the mean line. In metal AM, the surface roughness of the deposited part could be affected by the point distance, hatch distance, surface balling, staircase effect, and powder size distribution, for example. By isolating some parameters, certain parameters may be revealed from the surface finish as follows.

(1) Point distance: Figure 1 shows the point distance of a powder bed AM process. For the AM process with a pulse laser, the point distance is the distance between two pulses. When the point distance is small, similar to a continuous laser, the surface roughness can be improved. Increases in point distance lead to increased surface roughness. In a $316 \mathrm{~L}$ powder bed process, the surface roughness was primarily affected by point distance, with increased point distance resulting in increased surface roughness $(R a)$ from 10 to $16 \mu \mathrm{m} .{ }^{(10)}$

(2) Hatch distance: Figure 1 also shows the hatch distance of an AM process. Hatch distance can determine the overlap percentage between two deposition tracks. Figure 2 illustrates the impact of hatch distance on surface roughness. ${ }^{(11)}$ When the hatch distance is large, as shown in Fig. 2(a), there may be a cavity between two tracks. Thus, the surface may be rough. When the hatch distance is reduced to the proper distance shown in Fig. 2(b), the cavity may be greatly reduced to nearly flat. Thus, the surface may be smooth. When the hatch distance is further reduced, as shown in Fig. 2(c), the material may accumulate as a lump. Thus, the overall surface may become rough again.

(3) Surface balling: During the metal deposition, precise process control is needed. The high temperature gradients and densification ratio during the process yield the risk of balling formation in the melt pool, thereby resulting in a rough deposited surface. However, the

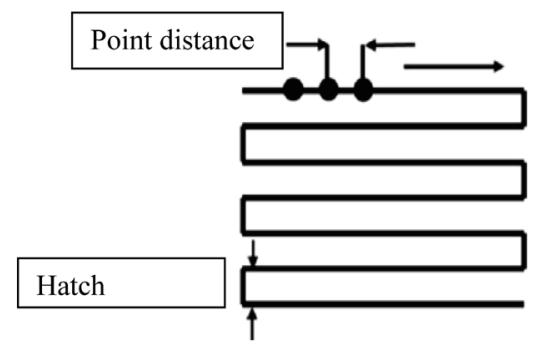

Fig. 1. Deposition tool path of a pulse laser in a powder bed AM process. ${ }^{(1)}$

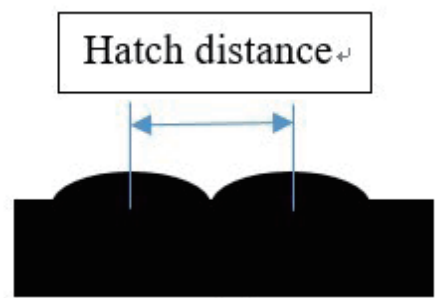

(a)

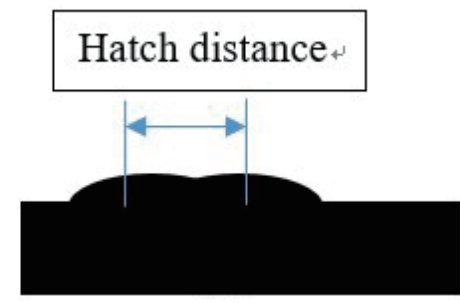

(b)

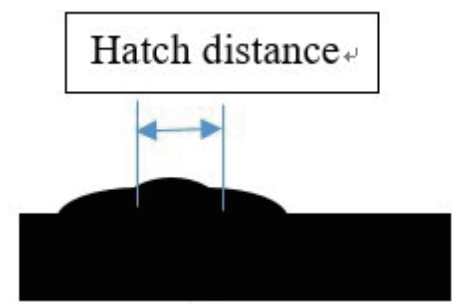

(c)

Fig, 2. (Color online) Illustration of effect of various hatch distances on surface roughness. 
adverse effect of low temperature may result in breaking up the liquid scan track during deposition and produce particles of spherical shapes. Thus, surface balling is apparent at both low and high laser energy densities. Both cases will result in a rough surface finish.

(4) Staircase effect: As shown in Fig. 3, the large thickness of slices in AM often produce rough surface quality owing to the staircase effect on the side surface of the component.

(5) Powder size distribution: Powder particles of various sizes, especially fine particles, are needed to optimize the part properties, such as part density, surface roughness, and mechanical strength. ${ }^{(3)}$ Similar results have been reported, that is, a fine powder granulation generally leads to better density and surface qualities than does a coarse material. ${ }^{(12)}$

\section{Hardness Test}

Hardness is the degree of how resistant a solid material is to shape change when a compressive force is applied. Many hardness tests exist, in which the material is indented until an impression is formed. These tests can be performed on a macroscopic or microscopic scale. It is a local material property and is typically not considered as a fundamental material property. However, since hardness is dependent on many other material properties, such as elastic stiffness, plasticity, strength, ductility, strain, toughness, viscoelasticity, and viscosity, if measured by a proper procedure, it may reveal much material information. The surface finish of an object does not have an effect on the hardness measurement if only the indentation is larger than the surface roughness. Since the hardness test is a nondestructive evaluation method, it can provide a very effective and economical first assessment of the material properties in AM. In general, hardness is related to microstructure, composition, grain size, and porosity.

(1) Material composition

Hardness is related to material composition. Thus, each material reveals a different hardness value. For example, direct fusion joining between two alloys, titanium alloys, and stainless steel can cause brittle Ti-Fe intermetallics, which compromise the mechanical properties of diffusion bonds. Vickers hardness tests were conducted for materials from $\mathrm{Ti}_{6} \mathrm{Al}_{4} \mathrm{~V}$ to $\mathrm{SS} 316$. The result of this direct metal deposition study is shown in Fig. 4. The hardness of the near-interface region is much higher than that of the base alloys on both sides. It is demonstrated that the compounds near the crack region have poor plasticity. During deposition, a crack is generated just under the action of a very small thermal stress. From the hardness distribution in Fig. $4{ }^{(13)}$

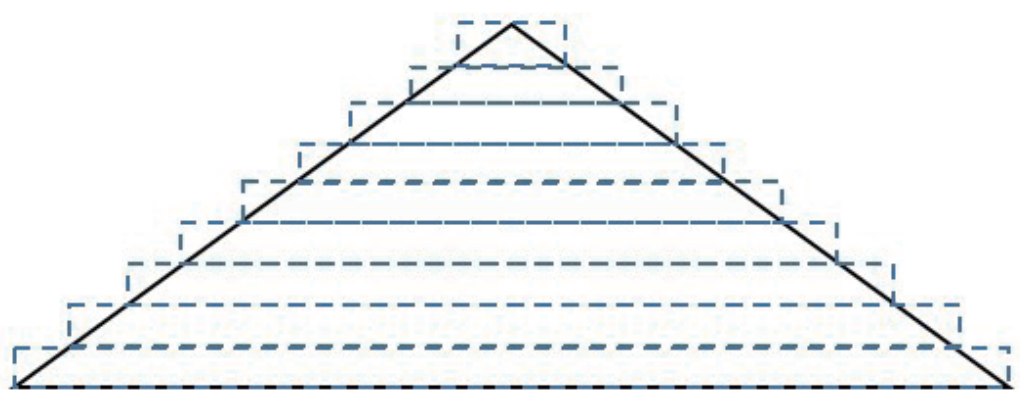

Fig. 3. (Color online) Staircase effect on side surface roughness of the component. 


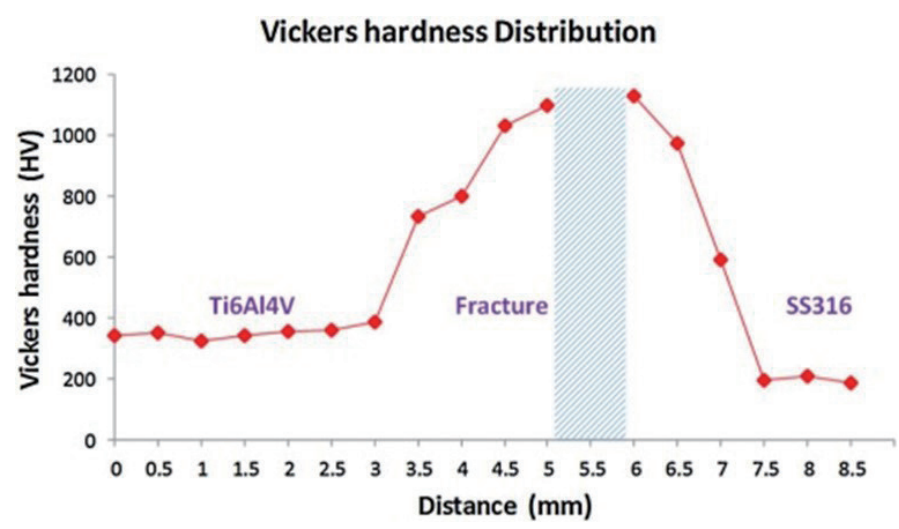

Fig.4. (Color online) Vickers hardness distribution along the deposition of $\mathrm{Ti}_{6} \mathrm{Al}_{4} \mathrm{~V}$ and $\mathrm{SS} 316 .{ }^{(14)}$

we found that hardness remains approximately constant for the $\mathrm{Ti}_{6} \mathrm{Al}_{4} \mathrm{~V}$ substrate, then starts to increase close to the crack region, and reaches the maximum of 1130 Vickers hardness number (VHN). The perforative crack appeared at this location. The hardness distribution in Fig. 4 illustrates that the formation of intermetallic phases is the primary cause of the failure when stainless-steel powder is laser-deposited directly on a titanium alloy substrate. This is a good example of using hardness to reveal parts of varying compositions.

(2) Grain size

Resistance to plastic deformation is an important grain-size-related property of metals. One of the simple measures of this resistance is the hardness test. The Hall-Petch relationship ${ }^{(14,15)}$ describes the linear relationship between the hardness $H$ and the grain size $d$ as

$$
H=H_{0}+K H d^{-\frac{1}{2}}
$$

Here, $H_{0}$ and $\mathrm{KH}$ are hardness constants. This equation expresses the fact that the finer the grain, the harder the material, unless the grain size is much smaller (less than $10 \mathrm{~nm}$ or so).

It can be used to predict the properties of several different materials, such as metals, ceramics, intermetallics, and polymers. There is a direct grain size effect on ductile fracture, although there is certainly an indirect effect of grain size on strength.

Since yield strength is proportionally related to hardness, the Hall-Petch equation has been expressed in terms of yield strength as ${ }^{(16)}$

$$
\sigma=\sigma_{0}+K y d^{-\frac{1}{2}}
$$

where $\sigma_{0}$ and $K y$ are yield constants.

Since grain size will also affect other strengths, such as fatigue, the fatigue strength is often treated as varying with grain size in the same manner. ${ }^{(16)}$ 
Another important factor in the metal deposition process is the cooling rate. The greater the temperature difference, the quicker the phase transformation tends to occur. Thus, a higher cooling rate will result in finer grains.

During metal AM process development, often an optimal set of process parameters is needed. In this case, the input powder material is very similar. Thus, the composition is similar between batches. Therefore, the hardness of the deposited samples, if strategically measured, can be used in the first screening of the quality of the deposited samples. Harder material samples could result from smaller grain size or higher density.

Figure 5 shows the variation of deposition microhardness with scan speed for 316L stainless steel laser-deposited directly on ASTM 4340 steel with a (1) laser power of $940 \mathrm{~W}$ and powder flow rate of $3.6 \mathrm{gpm}$; (2) laser power of $760 \mathrm{~W}$ and powder flow rate of $3.6 \mathrm{gpm}$; and (3) laser power of $760 \mathrm{~W}$, powder flow rate of $5.3 \mathrm{gpm}$. It is relevant that the fabricated deposition hardness increases with increasing scan speed. This is mainly due to the short interaction time between the laser and the powder. A comparison of the plots in Fig. 5 shows that, in the range of the presented parameters, when the powder flow rate decreases, the deposition hardness increases. In the single-layer deposition, the high cooling rate may be attributed to the high surface-to-volume ratio. The high cooling rate can cause the refinement of the grain size, thus increasing the deposition hardness. It could be concluded that in the presented range of laser cladding parameters, a higher scan speed and a lower powder flow rate should be chosen for improved hardness.

(3) Porosity or density

Since the hardness is an indicator of resistance to plastic deformation, it is closely related to the porosity or density of the material. In metal deposition, porosity generation is mainly driven by two factors, the gas trapped during solidification and the lack of fusion. Total porosity is found to be a strong function of laser energy density. At low laser energy density, porosity

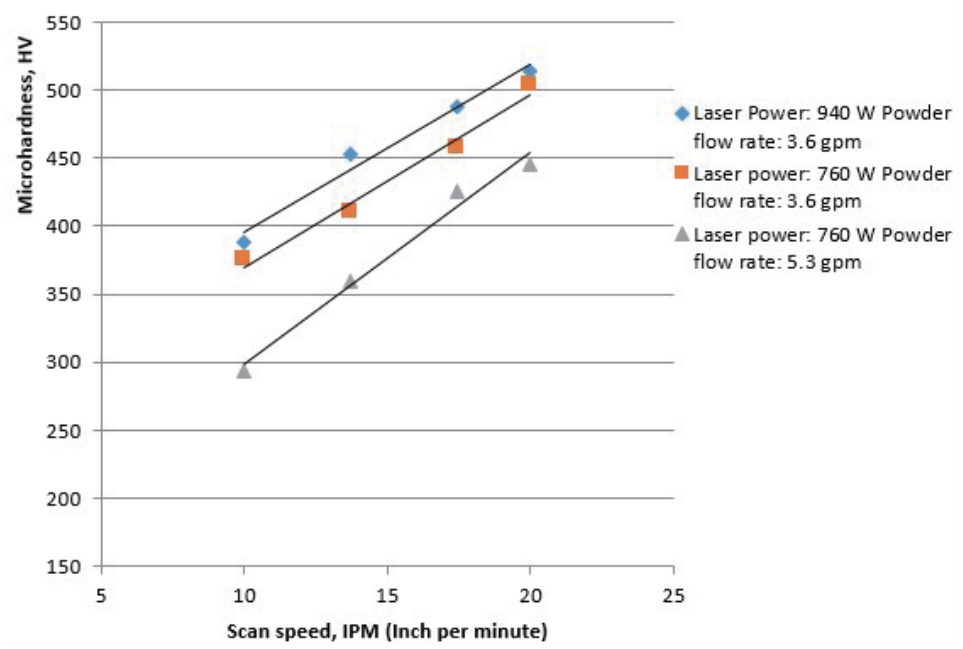

Fig. 5. (Color online) Variation of microhardness of laser-deposited 316L stainless steel on ASTM 4340 steel with scan speed. 
is high and decreases as laser energy density increases, only to increase again with further increase in the laser energy density. If the solidification velocity of the melt pool is higher than the gas exit velocity, gas can become trapped inside the deposited material. The lack of fusion might be due to insufficient power to melt the powder or too much powder. In general, the porosity causes the hardness, tensile strength, and fatigue strength to decrease. ${ }^{(17,18)}$ In other research, ${ }^{(10)}$ material hardness reached a maximum of $225 \mathrm{HV}$ at $125 \mathrm{~J} / \mathrm{mm}^{3}$ and was related to the material porosity, with increased porosity leading to decreased material hardness.

\section{Discussion}

Both hardness and surface roughness tests are tests of local material properties. However, since they are nondestructive testing methods, if properly planned, they could be used to reveal some important information in metal AM. In other words, if hardness and surface roughness tests can be performed to cover most of the samples, they could be used for the initial assessment of the samples during a similar metal deposition process before destructive methods, such as tensile or fatigue tests, are carried out.

For example, if the same material is used for an AM process and only some process parameters, such as laser power and scan speed, are varied in the processing, the hardness tests can reveal which process parameters can yield finer grain size and/or higher density. This is because the material composition can be assumed to remain the same. The surface roughness tests may show the differences between batches in the surface balling effect, since the rest of the factors, such as the point distance, hatch distance, staircase effect, and powder size distribution can be assumed to be similar. If the standard or optimal point distance, hatch distance, and powder size distribution for a specific deposition can be used in testing the scan surface of the sample, then the surface finish can provide more in-depth information about the balling effect. When the energy density is too low or too high, the surface balling effect is likely to be in effect. Thus, it is feasible to use this strategy to optimize the parameters to reduce surface roughness. Once the basic parameters are optimized, more process variables can be applied, and both the hardness and surface roughness can be used to conduct an initial assessment.

\section{Conclusions}

In this paper, we proposed an initial testing strategy for parts produced by metal AM processes. The apparent part properties, such as surface roughness, density, and hardness, can be used for the initial assessment of the deposited part. From the first-order estimation, the surface roughness, density, and hardness of the part are related to some key process parameters, such as energy density and track overlap. In addition, the part hardness is related to the cooling rate during AM processing, the part density is related to powder packing density, and the surface roughness is related to powder grain size. In this paper, we described the interrelationships among various parameters. Depending on the application, the identified parameters can be used for online or offline inspections using various sensor technologies. With the knowledge gained from this work, by using basic sensors, such as a microhardness tester and profilometer, critical information about an additive manufactured part can be obtained can be obtained. 


\section{Acknowledgments}

The authors would like to thank the Ministry of Science and Technology (MOST) and Fisheries Agency, Council of Agriculture (FA.COA) for their support of the project [Grant Nos. MOST 107-2218-E-006-031-, MOST 107-2218-E-110-004-, and 107AS-14.2.7-FA-F1(3)]. Additionally, this research was, in part, supported by the Ministry of Education, Taiwan, Headquarters of Advancement to the Intelligent Manufacturing Center (iMRC), National Cheng Kung University (NCKU), and US National Science Foundation (CMMI 1625736).

\section{References}

1 Wohlers Associates Industry Briefing: https://wohlersassociates.com/brief02-13.html (accessed February 2013).

2 M. Averyanova and P. Bertrand: Int. Conf. Advanced Research in Virtual and Rapid Prototyping (CRC Press, Leiria, 2010).

3 A. B. Spierings, N. Herres, and G. Levy: Rapid Prototyping J. 17 (2011) 195. https://doi. org/10.1108/13552541111124770

4 A. Berkau: Einsatzmöglichkeiten des Strahlschmelzens zur Herstellung von Bauteilen aus Aluminium, in 16. Anwenderforum Rapid Product Development (citim GmbH, Stuttgart, 2011) (in German).

5 E. Brandl, U. Heckenberger, V. Holzinger, and D. Buchbinder: Mater. Des. 34 (2012) 159. https://doi. org/10.1016/j.matdes.2011.07.067

6 D. Buchbinder, H. Schleifenbaum, S. Heidrich, W. Meiners, and J. Bültmann: Phys. Procedia 12 (2011) 271. https://doi.org/10.1016/j.phpro.2011.03.035

7 L. E. Murr, S. A. Quinones, S. M. Gaytan, M. I. Lopez, A. Rodela, E. Y. Martinez, D. H. Hernandez, E. Martinez, F. Medina, and R. B. Wicker: J. Mech. Behav. Biomed. Mater. 2 (2009) 20. https://doi.org/10.1016/ j.jmbbm.2008.05.004

8 K. A. Mumtaz, P. Erasenthiran, and N. Hopkinson: J. Mater. Process. Technol. 195 (2008) 77. https://doi. org/10.1016/j.jmatprotec.2007.04.117

9 L. Rickenbacher, T. Etter, S. Hövel, and K. Wegener: Rapid Prototyping J. 19 (2013) 282. https://doi. org $/ 10.1108 / 13552541311323281$

10 J. A. Cherry, H. M. Davies, S. Mehmood, N. P. Lavery, S. G. R. Brown, and J. Sienz: Int. J. Adv. Manuf. Technol. 76 (2015) 869. https://doi:10.1007/s00170-014-6297-2

11 T. Trosch, J. Strößner, R. Völkl, and U. Glatzel: Mater. Lett. 164 (2016) 428. https://doi.org/10.1016/ j.matlet.2015.10.136

12 M. Badrosamay, E. Yasa, J. Van Vaerenbergh, and J. P. Kruth: Tech. Pap. Soc. Manuf. Eng. TP09PUB17 (2009).

13 W. Li, L. Yan, S. Karnati, F. Liou, J. Newkirk, K. Taminger, and W. Seufzer: J. Mater. Process. Technol. 242 (2017) 39. https://doi.org/10.1016/j.jmatprotec.2016.11.010

14 E. O. Hall: Proc. Phys. Soc. London, Sect. B (1951) 742. https://doi.org/10.1088/0370-1301/64/9/302

15 N. J. Petch: J. Iron Steel Inst. 174 (1953) 25.

16 J. W. Morris: The Influence of Grain Size on the Mechanical Properties of Steel (2001). http://escholarship.org/ uc/item/88g8n6f8

17 N. Kurgan: Mater. Des. 55 (2014) 235. https://doi.org/10.1016/j.matdes.2013.09.058

18 J. Luo and R. Stevens: Ceram. Int. 25 (1999) 281. https://doi.org/10.1016/S0272-8842(98)00037-6

\section{About the Authors}

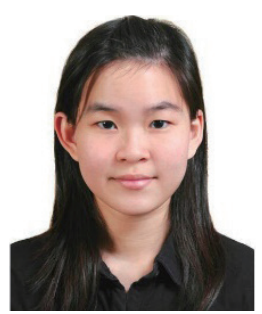

Yi-Chien Cheng received her B.S. degree in electrical engineering from National Changhua University of Education, Taiwan, in 2017. She is currently working toward her M.S. degree in the Department of Systems and Naval Mechatronic Engineering, National Cheng Kung University, Tainan, Taiwan. Her research is on additive manufacturing (AM) under the supervision of Prof. Frank Liou. Because of her electrical engineering background, her research interests also include power systems and electric machinery. (zxc8069@yahoo.com.tw) 


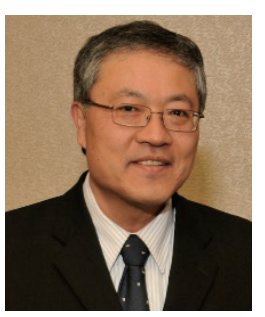

Frank Liou is the Michael and Joyce Bytnar Professor of the Mechanical Engineering Department of Missouri University of Science and Technology. He has served as the Director of the Manufacturing Engineering Program at Missouri S\&T since 1999. He has published a book on Rapid Prototyping along with over 300 technical papers. Dr. Liou's research focuses on additive manufacturing (AM), including hybrid additive and subtractive processes, path planning, multiscale multiphysics process modeling, and AM process monitoring and control. His research has been funded by AFRL, DOE, NASA, NAVY, NSF, and many industrial partners. Dr. Liou has received several teaching, research, and service awards, including several best paper awards. Dr. Liou is a Fellow of ASME. (liou@mst.edu)

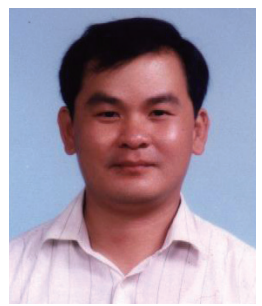

Chih-Kun Cheng was born in 1963 in Tainan, Taiwan. He received his B.S. degree in industrial education in 1987 from National Taiwan Normal University. He received his M.S. degree in electrical engineering from National Cheng Kung University in 1999, and presently, he is in the Ph.D. program of the Department of Electrical Engineering, National Cheng Kung University. At present, he is the chairperson of the Department of Electrical Engineering, Far East University, Tainan, Taiwan. He has joined the transformer research group since conducting his M.S. thesis research. His major interests are in transformer technology, power systems, and electric machinery. (n2888122@yahoo.com.tw)

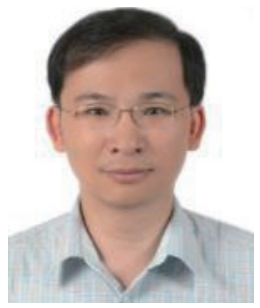

Sheng-Chih Shen received his B.E. and M.S. degrees in automatic control engineering from Feng Chia University, Taiwan, in 1996 and 1998, respectively. He received his Ph.D. degree in 2002 from the Department of Engineering and System Science, National Tsing Hua University, Hsinchu, Taiwan. He was a researcher in the MEMS division of the ITRI from 2002 to 2007, and a visiting scholar in the field of MEMS at Carnegie Mellon University from 2004 to 2005. He joined the Department of Systems and Naval Mechatronic Engineering, National Cheng Kung University, Tainan, Taiwan, as a professor in 2018. His current research interests focus on PVDF fiber sensors, LED fishing lighting, and underwater optic lighting. (scshen@mail.ncku.edu.tw) 